\title{
An All-Organic Photochemical Magnetic Switch with Bistable Spin States
}

\author{
Konstantin Günther ${ }^{\ddagger}$ Niklas Grabicki, ${ }^{\ddagger}$ Beatrice Battistella, Lutz Grubert, and Oliver Dumele* \\ Department of Chemistry \& IRIS Adlershof, Humboldt University of Berlin, Brook-Taylor-Strasse 2, D-12489 Berlin, \\ Germany
}

\begin{abstract}
Controlling the electronic spin state in single-molecules through an external stimulus is of interest in developing devices for information technology, such as data storage and quantum computing. We report the synthesis and operation mode of two all-organic molecular spin state switches that can be photochemically switched from a diamagnetic (EPR silent) to a paramagnetic (EPR active) form at cryogenic temperatures due to a reversible electrocyclic reaction of its carbon skeleton. Facile synthetic substitution of a configurationally stable 1,14-dimethyl-[5]helicene with radical stabilizing groups at the 4,11-positions afforded two spin state switches as 4,11-dioxo or

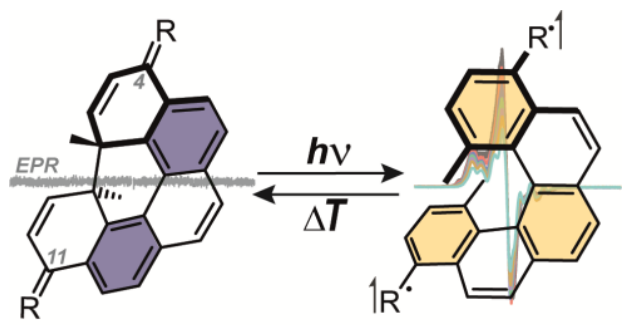
4,11-bis(dicyanomethylidenyl) derivatives in a closed diamagnetic form. After irradiation with an LED light source at cryogenic temperatures a stable paramagnetic state is readily obtained, rendering this system a bistable magnetic switch that can reversibly react back to its diamagnetic form through a thermal stimulus. The switching can be monitored with UV/Vis spectroscopy, EPR spectroscopy, or induced by electrochemical reduction and reoxidation. Variable-temperature EPR spectroscopy of the paramagnetic species revealed an open-shell triplet ground state with an experimentally determined triplet-singlet energy gap of $\Delta E_{\mathrm{T}-\mathrm{S}}<0.1 \mathrm{kcal} \mathrm{mol}^{-1}$. The inherent chirality and the ability to separate the enantiomers turns this helical motif into a potential chiroptical spin state switch. The herein developed 4,11-substitution pattern on the dimethyl[5] helicene introduces a platform for designing future generations of organic molecular photomagnetic switches that might find applications in spintronics and related fields.
\end{abstract}

\section{INTRODUCTION}

Modern information technology (IT) relies on the ability to store and process large amounts of data within a relatively short amount of time. This process is based on a controlled change of a particular physical property, such as the electrical, magnetic, or optical response of an information bit. ${ }^{1-4}$ That degree of control requires materials with at least two distinct states that can be altered by an external stimulus. In an ongoing demand for miniaturization of IT devices the search for the smallest possible information storing and processing unit has brought the attention towards single molecules. In this context, molecular organic photoswitches are an intensively studied field in which a particular property, such as basicity, catalytic or biological activity, mechanical and electric conducting properties, can be switched with high spatiotemporal resolution using light as an external stimulus. ${ }^{5,6}$ Of particular interest for potential applications in IT devices as qubits ${ }^{7,8}$ and contrast agents is the external control over the spin state of isolated molecules that leads to magnetic bistability. Controlling the spin state of discrete molecules has been demonstrated for metalorganic spin-crossover complexes or photochromic complexes containing first-row transition metals such as nickel or cobalt. ${ }^{9-15}$

Successful switching of spin states in all-organic molecules is rare. ${ }^{16-20}$ It can be realized based on several molecular mechanisms, ${ }^{20}$ but only two concepts that rely either on 1) a photoconformational trap (Figure 1, top) or on 2) a photochemical reaction (Figure 1, bottom) have the potential of achieving true magnetic bistability. An early example of a photo-induced paramagnetic diradicaloid form with simultaneous conformational change that gave rise to a paramagnetic state was reported by Toda and Tanaka, but no magnetic bistability was achieved (Figure 1a). ${ }^{16}$ Within a few hours, the thermal back-reaction converted the electron paramagnetic resonance (EPR)active form reversibly into the diamagnetic form. In contrast, magnetic bistability could be demonstrated based on a photoconformational change of an overcrowded aromatic ene by Kubo and co-workers (Figure 1b). ${ }^{18}$ The photo-generated paramagnetic form could be trapped in a least-strained orthogonal conformation with a thermal back-reaction occurring gradually at room temperature to revert the equilibrium into the diamagnetic form. In a subsequent optimization of Kubo's system, Feringa and coworkers introduced additional steric bulk in the fjordregion and thiaxanthylidene units that stabilize the diradical state, which eventually lead to absolute magnetic bistability at room temperature. However, the stabilization of spin states states that is dominantly based on conformational changes could be a conceptual limitation for the future demand of tuned bistability of molecular magnetic switches. Hence, switching mechanisms based on photochemical reactions that involve the making and breaking of chemical bonds is a motivating alternative concept to achieve amplified bistability among the magnetic states. 

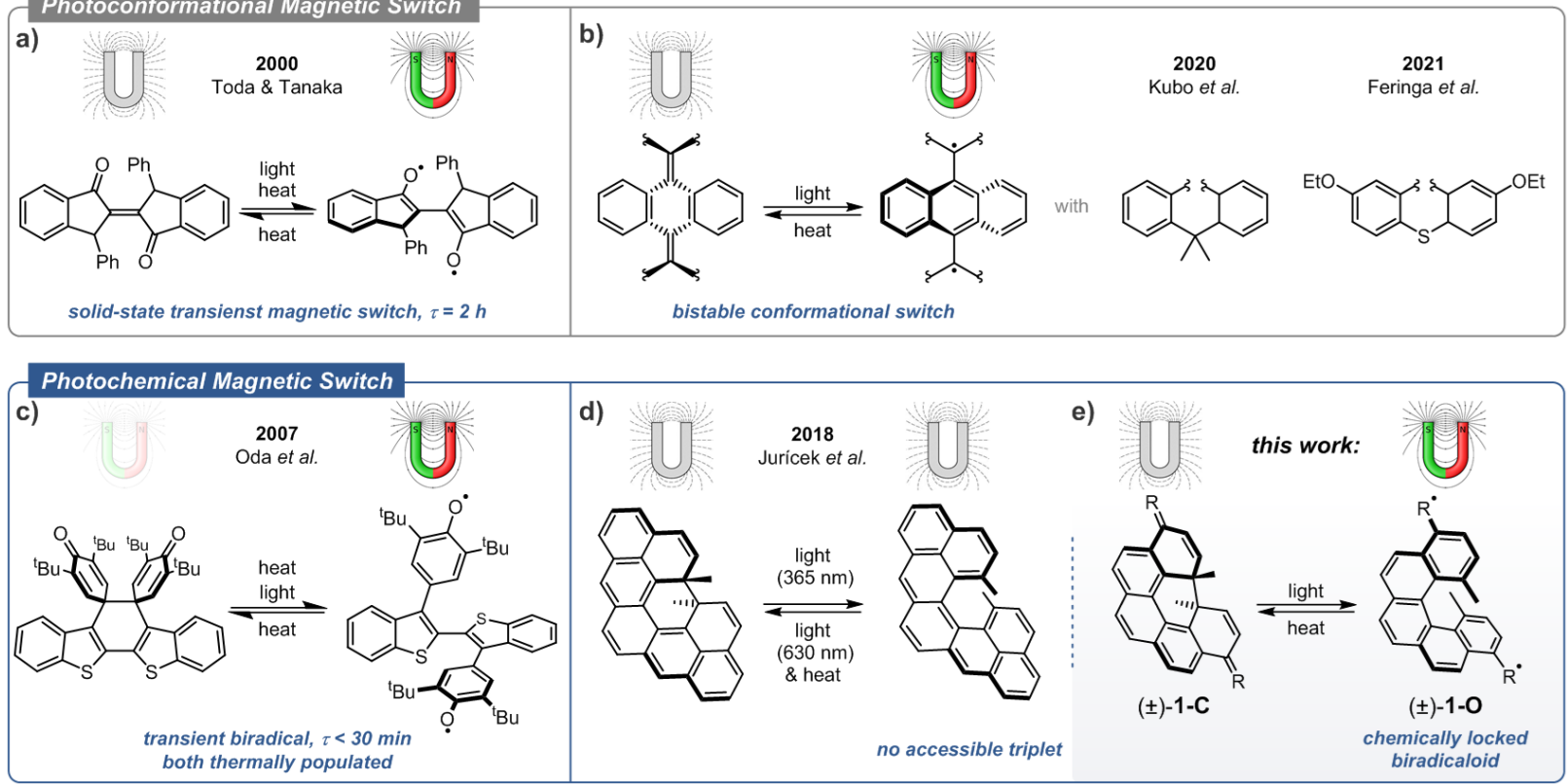

Figure 1. Photomagnetic molecular switches via conformational isomerization (top) or photochemical reactivity (bottom). Grey magnet: EPR-silent form; faint-colored magnet: weakly EPR-active form; colored magnet: EPR-active form.

In that context, the group of Oda has demonstrated the photochemical formal $6 \pi$ retroelectrocyclization of a bis(quinone-extended) thioindigo that partially increases the population of a diradicaloid form with a paramagnetic spin state (Figure 1c). ${ }^{17}$ Yet, only a minor ratio of paramagnetic-to-diamagnetic states was achieved due to the permanent thermal population of the triplet state in the dark. Juríček and co-workers introduced a novel photoswitchable dimethylcethrene with the promise of generating a diradicaloid triplet state upon irradiation of the closed diamagnetic form (Figure 1d). ${ }^{21}$ In their approach, a photochemical $6 \pi$ retroelectrocyclization gave rise to the open helical form containing a paraquinodimethane ( $p$-QDM) motif exhibiting an open-shell singlet biradicaloid structure. However, no population of the triplet state was found at room temperature because of a too high singlet-triplet energy gap ( $\Delta E_{\mathrm{ST}}$, calculated to ca. $10 \mathrm{kcal} \mathrm{mol}^{-1}$ for the open form). Hence, neither form showed a paramagnetic resonance signal using EPR spectroscopy.

Inspired by this approach of Juríček and co-workers, we herein present the first all-organic photochemical magnetic switch that shows bistability between two spin states upon breaking and making of chemical bonds (Figure 1e). We focused on a molecular design that stabilizes a diradicaloid form in the open [5] helicene 1-0 at the exocyclic 4,11positions by introducing radical-stabilizing groups $(\mathrm{O}$ or $\left.\mathrm{C}(\mathrm{CN})_{2}\right)$ that also accommodate a quinoidal closed form 1-C. By selecting this 4,11-substitution pattern, we sought after stabilizing the positions of highest spin density in a hypothetical biradical state of 1-0.

\section{RESULTS AND DISCUSSION}

Synthesis. Dibromo[5]helicene $( \pm)-3$ was synthesized in three reaction steps from commercially available starting materials following a previous report by Juríček and coworkers (see the Supporting Information Section S2). ${ }^{22}$ Palladium-catalyzed Miyaura borylation of dibromide $( \pm)-3$ gave bis(boronic acid pinacol ester) $( \pm)-2$ in good yields (74\%, Scheme 1). ${ }^{23}$ Subsequent oxidative deborylation of $( \pm)-2$ with an excess of hydrogen peroxide leads directly to diketone $( \pm)$-1a-C. Only the closed diketone is observed in the synthesis that is presumably obtained through a diradical mechanism upon oxidation of a transient open diol.24, 25 Closed bis(dicyanomethylidene) ( \pm )-1b-C was synthesized through a Pd-catalyzed Takahashi-coupling 26 from dibromide $( \pm)$-3 via dihydro intermediate $( \pm)-\mathbf{1 b}-\mathbf{0}$ $\mathbf{H}_{2}$, that underwent subsequent oxidation upon deprotonation and formal electrocyclization to give $( \pm)$-1b-C. Furthermore, we were able to separate the $(M)$ and $(P)$-enantiomers of boronic ester $( \pm)$-2 via chiral high performance liquid chromatography (HPLC) and the absolute configuration was validated using a combination of time-dependent density functional theory (TD-DFT) calculations, optical rotatory dispersion (ORD), and electronic circular dichroism (ECD) spectroscopy (see the Supporting Information Section S3, S4). Unambiguous proof of the absolute configuration was obtained by a singlecrystal X-ray structure of enantiopure $(+)-(P)-2$ (Figure 2). The pure enantiomers of bis(boronate) $(P / M)-2$ could be oxidized separately to give enantiopure stereoisomers (-)$(R, R)-\mathbf{1 b}-\mathbf{C}$ and $(+)-(S, S)-\mathbf{1 b}-\mathbf{C}$. That strategy enables rapid access to enantiopure dimethyl[5]helicene derivatives, which can be applied as switches with chiroptical properties in the future. 
Scheme 1. Synthesis of $( \pm)-\mathbf{1 a}-\mathrm{C}$ and $( \pm)-\mathbf{1 b}-\mathrm{C}^{a}$

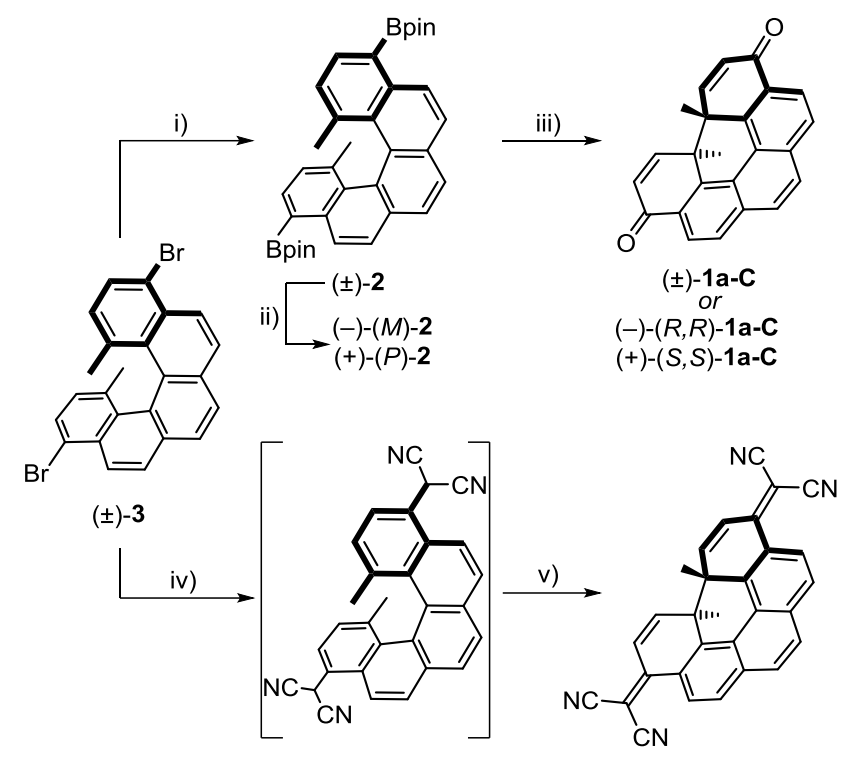

$( \pm)-1 b-0-H_{2}$
${ }^{a}$ Reagents and conditions: i) $( \pm)-3 \quad(1.0 \quad$ eq. $)$, bis(pinacolato)diboron (2.2 eq.), $\left[\mathrm{Pd}(\mathrm{dppf}) \mathrm{Cl}_{2}\right] \cdot \mathrm{CH}_{2} \mathrm{Cl}_{2} \quad(10$ mol\%), KOAc (5.0 eq.), 1,4-dioxane, $80{ }^{\circ} \mathrm{C}, 3.5 \mathrm{~h}, 74 \%$; ii) enantiomer separation by HPLC on a chiral stationary phase; iii) for $( \pm)-\mathbf{1 a}-\mathrm{C}:( \pm)-2\left(1.0\right.$ eq.), $\mathrm{H}_{2} \mathrm{O}_{2}\left(25.0\right.$ eq.), THF, $25^{\circ} \mathrm{C}, 6 \mathrm{~h}$, 78\%; $(+)-(S, S)-\mathbf{1 b}-\mathbf{C}(70 \%)$ from $(+)-(P)-2$ and $(-)-(R, R)-\mathbf{1 b}-\mathbf{C}$ $(63 \%)$ from (-)-(M)-2; iv) (1) malononitrile (6.0 eq.), $\mathrm{NaH}(10.0$ eq.), THF, $0{ }^{\circ} \mathrm{C}, 0.5 \mathrm{~h}$; (2) $( \pm)-3$ (1.0 eq), $\left[\mathrm{Pd}\left(\mathrm{PPh}_{3}\right)_{2} \mathrm{Cl}_{2}\right]$ (10 mol\%), [Pd(PPh $\left.)_{4}\right]$ (10 mol\%), THF, $80{ }^{\circ} \mathrm{C}, 16 \mathrm{~h}$, not isolated; v) $\mathrm{CHCl}_{3}, \mathrm{NEt}_{3}$, air, $25{ }^{\circ} \mathrm{C}, 30 \%$; dppf $=1,1^{\prime}-$ bis(diphenylphosphino)ferrocene, pin = pinacolato.

All novel compounds were characterized by NMR spectroscopy, HRMS, and FT-IR spectroscopy. The closed helical structures of $( \pm)-\mathbf{1} \mathbf{a}-\mathbf{C}$ and $( \pm)-\mathbf{1 b}-\mathbf{C}$ were confirmed by ${ }^{1} \mathrm{H},{ }^{13} \mathrm{C}-\mathrm{HMBC}$ NMR spectroscopy showing ${ }^{3} \mathrm{~J}_{\mathrm{H}-\mathrm{C}}$ couplings between the protons of the 1,14-dimethyl groups and bridging carbon atoms (Figures S76 and S85). Unambiguous structural proof was obtained by singlecrystal X-ray structures for $( \pm)$-1a-C and $( \pm)-\mathbf{1 b}$-C (Figure 2) which both exhibit a $\mathrm{C}-\mathrm{C}$ distance of $1.57 \AA$ between the bridging quarternary carbon atoms. The helical pitch angles along the inner $\mathrm{sp}^{2}$-hybridized $\mathrm{C}-\mathrm{C}-\mathrm{C}-\mathrm{C}$ helices are $\left.6.15^{\circ}(( \pm)-1 \mathbf{a}-\mathbf{C})\right)$ and $\left.14.09^{\circ}(( \pm)-\mathbf{1 b}-\mathbf{C})\right)$, in conjunction with a nearly axial displacement of the methyl groups. The new compounds $\mathbf{1}(\mathbf{a}, \mathbf{b})-\mathbf{C}$ clearly show a quinoidal structure at the terminal benzannulated rings with pronounced alternating bond lengths when compared with the parent aromatic [5] helicene scaffold (Figure 2).

UV/Vis Spectroscopy. Intrigued by the fact that we could only isolate the closed form molecules $( \pm)-\mathbf{1 a}-\mathbf{C}$ and $( \pm)-\mathbf{1 b}$ C as earlier observed for dimethylcethrene, ${ }^{25}$ we assumed a photochemical $6 \pi$ retroelectrocyclization had led to a biradicaloid open form.

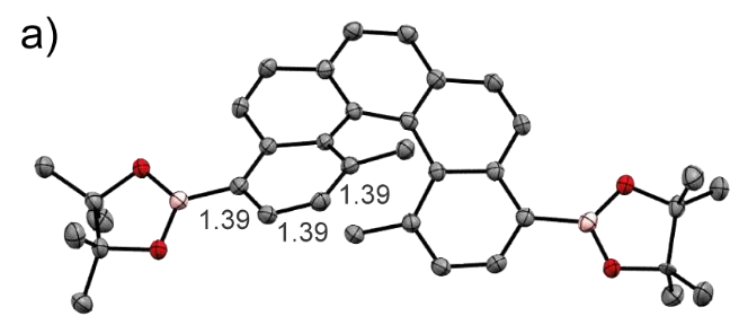

b)

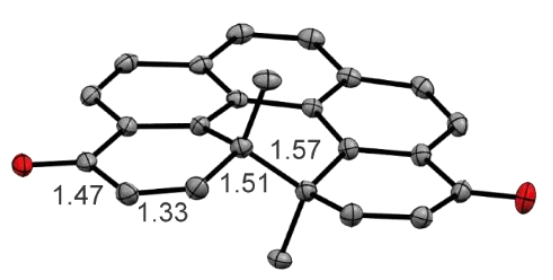

C)

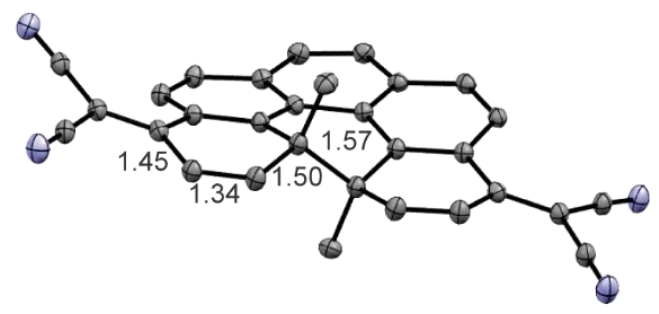

Figure 2. X-ray structures of a) $(+)-(P)-2$, b) $( \pm)-1 a-C$, and c) $( \pm)-\mathbf{1 b}-\mathbf{C}$. Hydrogen atoms are omitted for clarity and the thermal ellipsoids are shown at a $50 \%$ probability level (bond lengths in $\AA$ ).

We first investigated the optical absorption of these two compounds and found a hypsochromic shift of $( \pm)-\mathbf{1 a}-\mathbf{C}$ to $\lambda_{\max }=342 \mathrm{~nm}$ and $\mathrm{a}$ bathochromic shift of $( \pm)-\mathbf{1} \mathbf{b}-\mathbf{C}$ to $\lambda_{\max }=$ $414 \mathrm{~nm}$ compared to the open [5] helicene precursor $( \pm)-3$ $\left(\lambda_{\max }=362 \mathrm{~nm}\right)$ (Figure S7).

Initial irradiation of $( \pm)-\mathbf{1 a}-\mathbf{C}$ or $( \pm)-\mathbf{1 b} \mathbf{b}$ in $\mathrm{MeCN}$ with various wavelengths (LED light sources) at $295 \mathrm{~K}$ and above resulted in no observable change of the reaction mixture (see Figures S11, S16-S18). Assuming a fast thermal backreaction under these conditions, we turned to irradiation at cryogenic temperatures. Cooling to $77 \mathrm{~K}$ and irradiation of $( \pm)-\mathbf{1 a}-\mathbf{C}$ or $( \pm)-\mathbf{1 b}-\mathbf{C}$ in a glassy matrix of 2methyltetrahydrofuran (2-MTHF) with a $375 \mathrm{~nm}$ or $405 \mathrm{~nm}$ LED, respectively, resulted in significant changes of the UV/Vis absorption (Figure 3). After 13 min of irradiation for $( \pm)-\mathbf{1 a}-\mathbf{C}$ and 12 min for $( \pm)-\mathbf{1 b}-\mathbf{C}$ a photostationary state (PSS) is reached, which we assigned to a mixture of the open and closed forms $( \pm)-\mathbf{1 a}-\mathbf{C} /( \pm)-\mathbf{1 a}-\mathbf{0}$ or $( \pm)-\mathbf{1 b}-\mathbf{C} /( \pm)-\mathbf{1 b}-\mathbf{0}$. Both derivatives show distinct changes in the UV region of the spectra and several defined isosbestic points supporting a clean photochemical reaction. Several new broad absorption bands arise in the visible region at 400-550 nm for $( \pm)-\mathbf{1 a - 0}$ and $450-750 \mathrm{~nm}$ for $( \pm)-\mathbf{1 b}-\mathbf{0}$, indicative of a biradicaloid character of the open forms. 

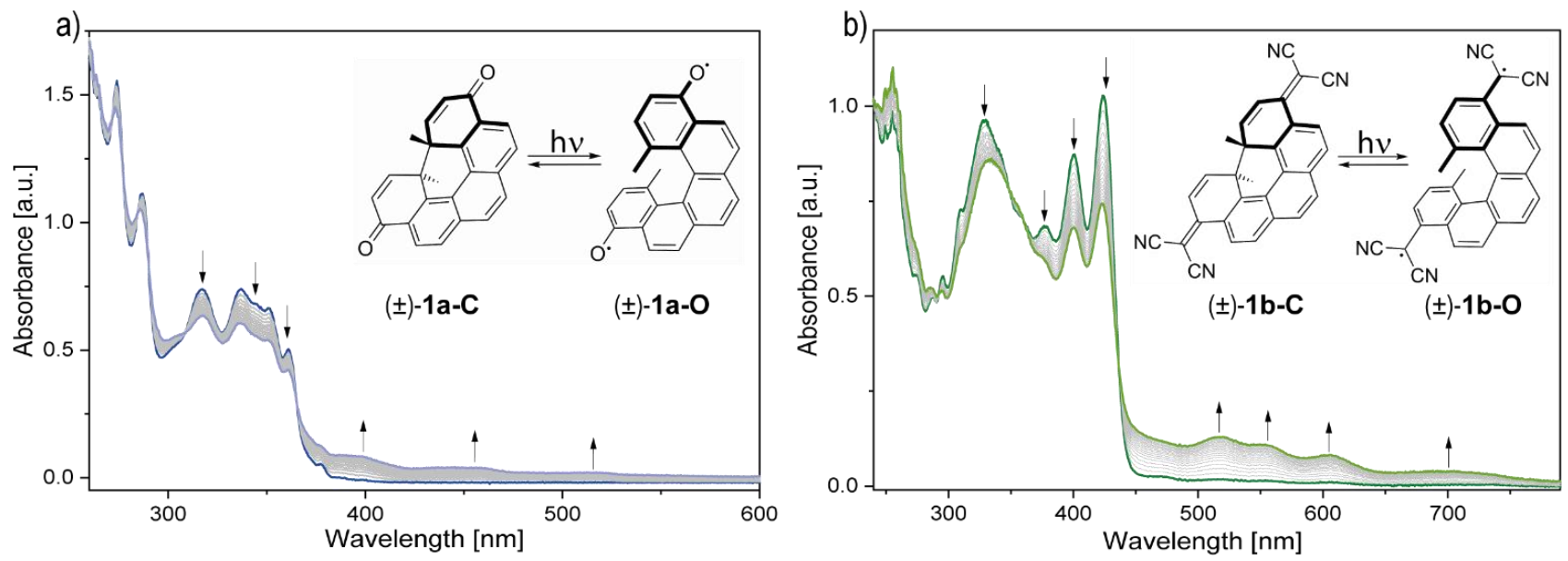

Figure 3. UV/Vis absorption spectra of a) $\left( \pm\right.$ )-1a (dark blue trace; $c=5.6 \times 10^{-5} \mathrm{M}$ ) irradiated with $375 \mathrm{~nm}$ light for $13 \mathrm{~min}$ (light blue trace) and b) ( \pm )-1b (dark green trace; $c=4.1 \times 10^{-5} \mathrm{M}$ ) irradiated with $405 \mathrm{~nm}$ light for 12 min (light green trace). All spectra were recorded in 2-MTHF at $77 \mathrm{~K}$; spectra shown as grey traces were recorded every $60 \mathrm{~s}$ during irradiation.

In both cases the photostationary state is retained for at least one hour at $77 \mathrm{~K}$ in the dark with no observable spectral changes (Figures S12, S19). While the photochemical back reaction of $( \pm)-\mathbf{1 a}-\mathbf{0}$ and $( \pm)-\mathbf{1 b}-\mathbf{C}$ at various tested wavelengths remained unsuccessful $(77 \mathrm{~K})$, heating above a critical temperature resulted in the complete disappearance of the newly formed absorption bands and the spectra of the closed form was recovered (Figures S13, S20). Detailed variable temperature (VTJUV/Vis absorption spectroscopy of the PSS $405 \mathrm{~nm}$ mixture with $( \pm)-\mathbf{1 b}$ revealed a rapid change of absorption bands at $92 \mathrm{~K}$, regenerating the spectrum of the closed form $( \pm)-\mathbf{1 b}$ C (Figures S21, S22). For the PSS $375 \mathrm{~nm}$ mixture of $( \pm)$-1a the absorption remained constant until the 2-MTHF matrix started melting at $127 \mathrm{~K}$ (Figure S14) and no defined critical temperature for the thermal ring closure of $( \pm)-\mathbf{1 a}-\mathbf{0}$ to $( \pm)$ 1a-C could be determined due to dominating scattering effects of the melting matrix. At temperatures of $140 \mathrm{~K}$, beyond the melting process of 2-MTHF, the thermal back reaction dominates again to form the closed form $( \pm)-\mathbf{1 a}-\mathbf{C}$ and irradiation $(405 \mathrm{~nm})$ at that temperature generated no $( \pm)$-1a-0.

EPR Spectroscopy. Successful switching encouraged us to investigate the EPR activity of $( \pm)-\mathbf{1 a}$ and $( \pm)$-1 $\mathbf{b}$ before and after irradiation in 2-MTHF at $77 \mathrm{~K}$. Before irradiation, the closed forms ( \pm )-1a-C and ( \pm )-1 b-C are EPR silent under the experimental conditions, but after irradiation for $10 \mathrm{~min}$ with $375 \mathrm{~nm}$ and $405 \mathrm{~nm}$ (LED), respectively, an intense EPR signal can be observed at $g \approx 2.0$ (Figure 4 and Supporting Information Figure S32). This process can be reversed upon heating the irradiated samples to the temperatures determined for the thermal back reaction from our previous VT-UV/Vis spectroscopy. Heating the PSS $_{375 \mathrm{~nm}}$ mixture of diketone $( \pm)-\mathbf{1 a}-\mathbf{C} / \mathbf{0}$ to room temperature immediately converted it to the pure closed form $( \pm)$-1a-C as confirmed by its EPR inactivity (77 K, second dark blue graph in Figure 4). Repetition of such a cycle including irradiation at $77 \mathrm{~K}$ and subsequent heating was performed up to three times with no observable degradation in the HPLC traces or EPR signal loss (Figure 4 and the Supporting Information Figure S29). The PSS $_{405 \mathrm{~nm}}$ mixture of bis(dicyano) ( \pm )-1 b-C/O rapidly converted back to the pure closed form $( \pm)-\mathbf{1 b}-\mathbf{C}$ upon heating to $93 \mathrm{~K}$ with the loss of the EPR signal, in agreement with our VT-UV/Vis study (Figure S32). From our experimental findings we conclude that diketone $( \pm)-\mathbf{1 a}-\mathbf{C}$ and bis(dicyanomethylidene) ( \pm )-1b-C can be photochemically switched into a stable EPR-active form and rapidly converted back to an EPR-silent form with a distinct thermal stimulus.

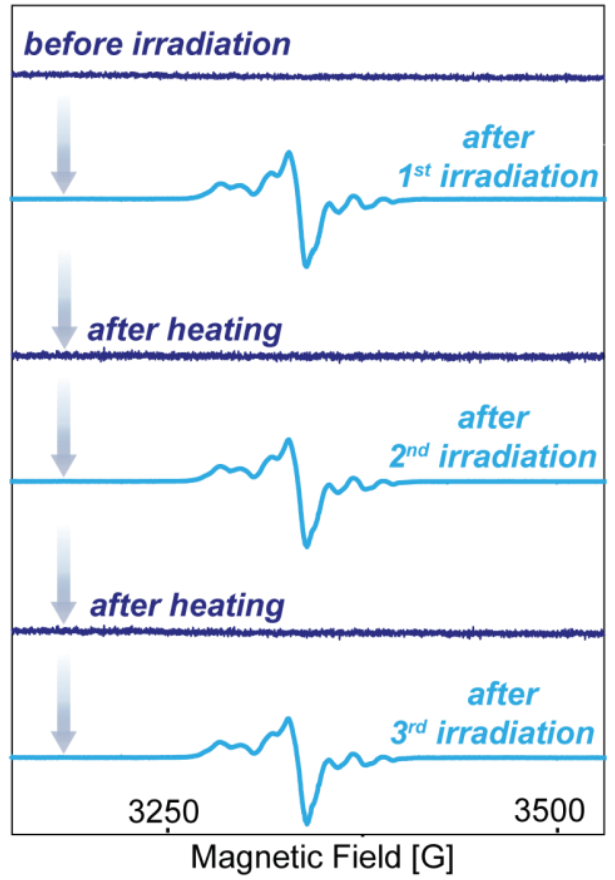

Figure 4. EPR spectra of three switching cycles of $( \pm)$-1a before and after irradiation with $375 \mathrm{~nm}$ for $10 \mathrm{~min}$ (light blue graphs, X-band EPR, $9.35 \mathrm{GHz}$, perpendicular mode, $0.5 \mathrm{~mW}, 1 \mathrm{G}$ modulation amplitude, $77 \mathrm{~K}$ ) and thermal back reaction through a heating loop to $295 \mathrm{~K}$ (dark blue graphs, X-band EPR, 9.35 GHz, perpendicular mode, $0.5 \mathrm{~mW}, 1 \mathrm{G}$ modulation amplitude, $13 \mathrm{~K}$ ).

In general, the singlet and triplet states in biradicaloids are thermally distributed with a distinct singlet-triplet energy 
gap ( $\left.\Delta E_{\text {ST }}\right)$. Thus, we performed VT-EPR spectroscopy of the irradiated open forms of $( \pm)$-1a and $( \pm)$-1 $\mathbf{b}$ and determined the spin multiplicity of their ground states. The EPR signal intensity of dioxo helicene $( \pm)-\mathbf{1 a - 0}$ measured under the same experimental conditions, decreases with increasing temperature from $13 \mathrm{~K}$ to $77 \mathrm{~K}$ representative of a triplet ground state (Figure 5). This ground state was further confirmed by our computational analysis (CASSCF active space $(8,8)$, vide infra). Non-linear curve fitting using the Bleaney-Bowers equation gave an experimental $\Delta E_{\mathrm{TS}}$ of $5.07 \times 10^{-2} \mathrm{kcal} \mathrm{mol}^{-1}$ (see the Supporting Information Figure S28). Under equal experimental conditions, the EPR intensity change with increasing temperatures of irradiated bis(dicyanomethylidene) $( \pm)-\mathbf{1 b}-\mathbf{0}$ is much smaller than that observed for the dioxo derivative $( \pm)-\mathbf{1 a - 0}$ and fitting to the Bleaney-Bowers equation is not possible (see the Supporting Information Figure S31).

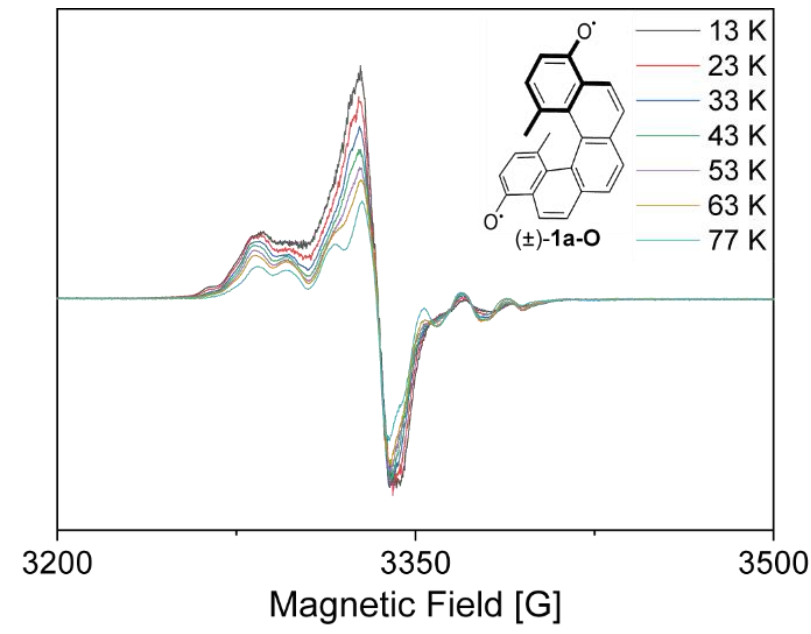

Figure 5. VT-EPR spectra of $( \pm)$-1a after irradiation (X-band EPR, 9.35 GHz, perpendicular mode, $0.5 \mathrm{~mW}, 1 \mathrm{G}$ modulation amplitude, $77 \mathrm{~K}$ ) for $10 \mathrm{~min}$ in 2-MTHF.

Theoretical Analysis. Bond breaking and making in this helicene switch proceeds through a formal $6 \pi$ electrocyclization reaction in a conrotatory fashion that is photochemically allowed but thermally forbidden. ${ }^{27}$ Juríček and co-workers have encountered a structurally similar thermal electrocyclization mechanism that is formally forbidden according to Woodward-Hoffman rules ${ }^{27}$ suggesting a diradicaloid mechanism. ${ }^{21,28}$

An analysis of the aromaticity based on resonance structures of the closed and open form allows to estimate whether an open- or closed-shell electronic configuration is favored. In the closed form 1-C, no diradicaloid character is expected due to the presence of two aromatic Clar's sextets and exocyclic double bonds to the quinoidal 4,11substituents ( $\mathbf{C}_{\mathbf{c s}}$, Scheme 2). ${ }^{21,29-32}$ In contrast, the open form has a major diradicaloid resonance contribution because of the presence of three Clar's sextets and localization of unpaired electrons at the exocyclic substituents ( $\mathbf{O}_{\mathrm{os}}$ ). The closed-shell resonance structure has an unfavorable all-quinoidal structure without localized aromatic Clar's sextets ( $\mathbf{O}_{\mathrm{cs}}$ ). Thus, it becomes obvious from this simple analysis that the open form-after irradiationhas a pronounced open-shell electronic configuration.
We further elaborate these considerations with the calculated energies of the open-shell and closed-shell structures for each helicene scaffold 1a,b (open and closed forms) using CASSCF calculations with an $(8,8)$ active space and the 6-31G(d,p) basis set (see Scheme 2). The energies of the closed form triplet states $\left(\mathbf{C}_{\mathbf{T}}\right)$ of $\mathbf{1 a}-\mathbf{C}_{\mathbf{T}}$ and $\mathbf{1} \mathbf{b}-\mathbf{C}_{\mathbf{T}}$ are significantly higher, by more than $50 \mathrm{kcal} \mathrm{mol}^{-1}$, than their closed-shell singlet states ( $\mathbf{C}_{\mathbf{c s}}$ ) thus supporting a pure closed-shell ground state before irradiation. The diradical character index $(y)$ of this ground state was calculated to 0.003 and 0.004 for $\mathbf{1} \mathbf{a}-\mathbf{C}$ and $\mathbf{1 b}-\mathbf{C}$, respectively, confirming a negligible diradical character (calculated by natural occupation orbital numbers analysis, see the Supporting Information Section S3). ${ }^{33}$ In contrast, the open-form helicenes have a triplet ground state $\left(\mathbf{0}_{\mathrm{T}}\right)$ with a calculated $\Delta E_{\mathrm{TS}, \text { calc }}$ of 5 and $8 \mathrm{kcal} \mathrm{mol}^{-1}$ for $\mathbf{1 a - 0}$ and for $\mathbf{1 b}-\mathbf{0}$, respectively. While this calculated value for $\Delta E_{\mathrm{TS}, 1 \mathrm{a}}$ is different compared to our experimentally determined $\Delta E_{\mathrm{TS}, 1 \mathrm{a}}$, the trend of the energy levels is retained. The biradicaloid character of the thermally accessible singlet state $\mathbf{O}_{\text {cs }}$ is enhanced for the open form which is represented by $y$ values of 0.12 and 0.84 for $\mathbf{1 a - 0}$ and $\mathbf{1 b}-\mathbf{0}$, respectively. Such ground state triplet diradicaloids are rare since the double spin-polarization effect turns the vast majority of diradicals into ground state singlets with a thermally accessible triplet state. ${ }^{34-41}$ However, a triplet ground state, as in our helical switch system, is desirable for obtaining a maximum response of the EPR active form.

Scheme 2. Resonance structures of the closed and open helicene forms of $\mathbf{1}^{a}$

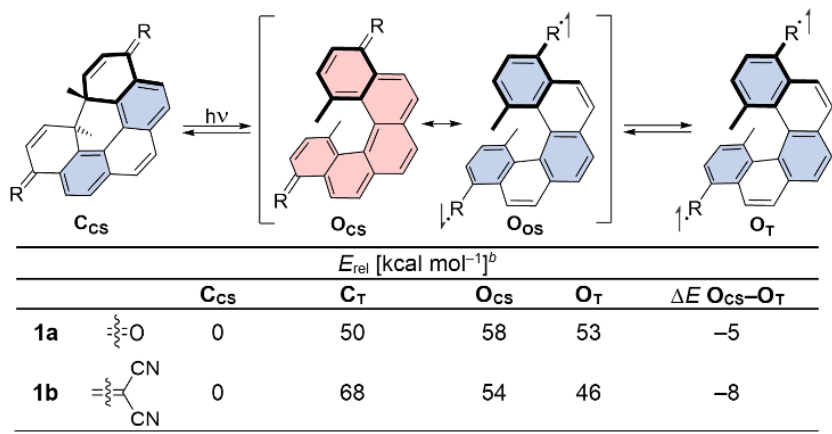

${ }^{a}$ Highlighted Clar's sextets in blue and quinoidal structures in red. ${ }^{b}$ Calculated energy values for $\mathbf{1 a}$ and $\mathbf{1 b}$ in their closed form as closed-shell singlet ( $\left.\mathbf{C}_{\mathbf{c s}}\right)$ and triplet $\left(\mathbf{C}_{\mathbf{T}}\right.$, not shown), and open forms as closed-shell singlet (0cs) and triplet $\left(\mathbf{O}_{\mathrm{T}}\right)$. Level of theory: CASSCF $(8,8) / 6-31 G(d, p)$.

Spectroelectrochemistry. To gain insight into possible electrochemical switching processes we turned to spectroelectrochemistry. Electrochemically initiated (retro)electrocyclization reactions have received much less attention compared to its photochemical equivalent. ${ }^{42-47}$ The few existing reports on electrochromic diarylethenes motivated us to investigate the redox properties of $( \pm)-\mathbf{1 a}$ and $( \pm)-\mathbf{1 b}$. We hypothesized that their corresponding radical anions or dianions should undergo ring-opening as the product would gain substantial stabilization due to 
aromatization compared to their closed forms. The cyclovoltammogram (CV) of diketone $( \pm)$-1a-C shows a reversible reduction at $E_{\mathrm{red}, 1}=-2.00 \mathrm{~V}\left(\mathrm{vs} . \mathrm{Fc} / \mathrm{Fc}^{+}\right.$, scan rate: $1 \mathrm{~V} \mathrm{~s}^{-1}$ ) and a second irreversible reduction at $E_{\mathrm{red}, 2}=-2.28$ $\mathrm{V}$. An additional anodic product peak at a potential of $-0.7 \mathrm{~V}$ is observed after sweeping towards a positive potential (Figure S36). We followed these electrochemical processes using in situ UV/Vis spectroscopy (scan rate: $10 \mathrm{mV} \mathrm{s}^{-1}$ ). Upon sweeping to the first reduction potential $E_{\text {red,1, the }}$ absorption profile changes with defined isosbestic points and a new absorption band arises between 700-900 nm (Figure 6a). This band can be assigned to the radical anion $( \pm)-1 a-C^{-}$, which is continuously produced and exists prior to the subsequent second reduction to give the open form dianion $( \pm)-1 \mathbf{1 a}^{-0^{2-}}$ with a new absorption maximum at 460 $\mathrm{nm}$ (for the detailed electrochemical mechanism, see the Supporting Information Schemes S1, S2). This is further supported by the comparison of the experimentally observed UV/Vis absorption spectrum of the reduced dianion and the spectra obtained from TD-DFT calculations for $\mathbf{1} \mathbf{a}-\mathbf{0}^{2-}$ (Figure S41, S42). Upon inverting the potential in $\mathrm{CV}$, the dianion $( \pm)-\mathbf{1 a - \mathbf { O } ^ { 2 - }}$ is stable up to an onset potential of $-1.0 \mathrm{~V} v s . \mathrm{Fc} / \mathrm{Fc}^{+}$where an anodic current is observed and
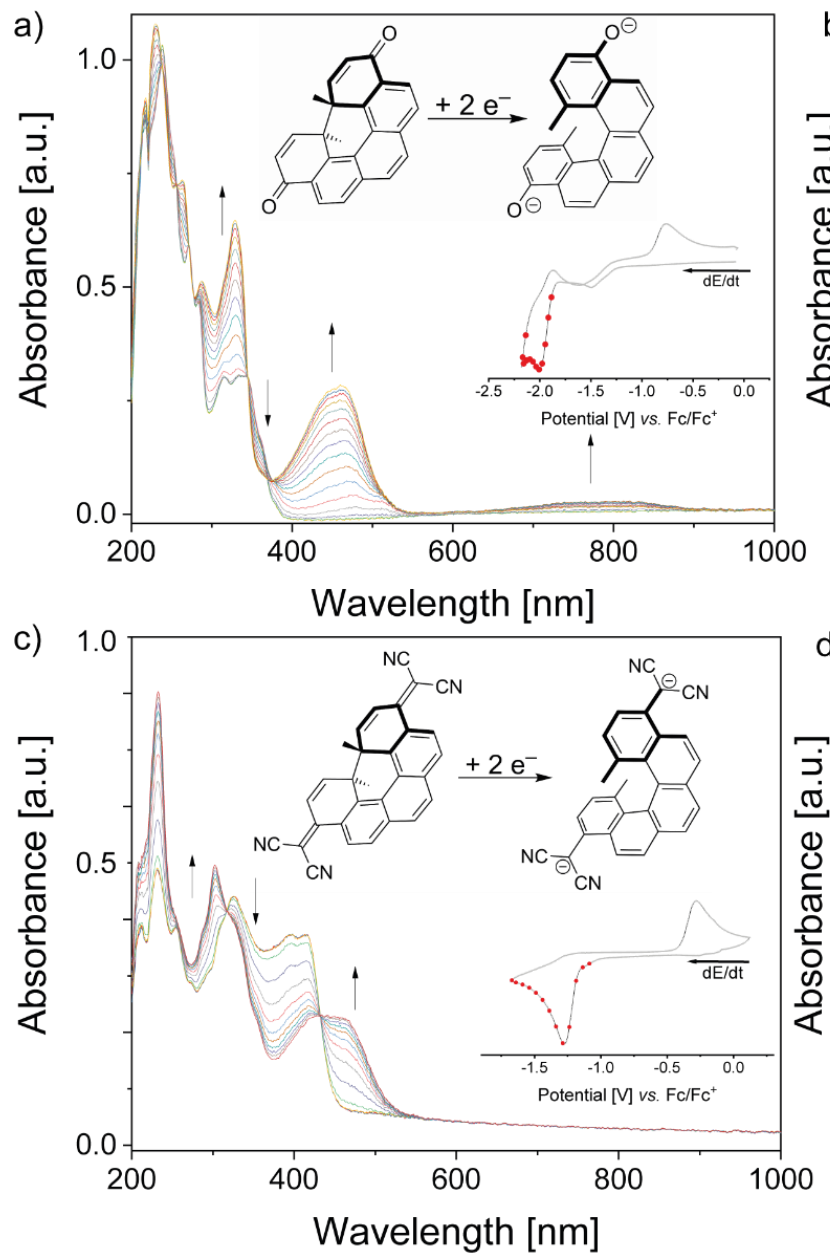

the intermediate is reoxidized to regain the closed form $( \pm)$-1a-C (Figure 6b).

Bis(dicyanomethylidene) $( \pm)-\mathbf{1 b}-\mathbf{C}$ shows an equivalent electrochemicaly induced switching. The CV trace shows one irreversible reduction wave at $E_{\text {red, } 1}=-1.32 \mathrm{~V} v s$. Fc $/ \mathrm{Fc}^{+}$ exclusively (scan rate: $1 \mathrm{~V} \mathrm{~s}^{-1}$ ), which we assign to a twoelectron transfer and the direct formation of the open dianion $( \pm)-\mathbf{1 b}^{2}-\mathbf{O}^{2}$ (Figure S43), again supported by the calculated TD-DFT spectra (Figure S47, S48). Here, the radical anion spontaneously opens to $\mathbf{1 b - \mathbf { O } ^ { - - }}$ and the second reduction, confirmed by quantitative electrolysis, occurs instantly under potential inversion conditions. ${ }^{48}$ The open dianion is stable up to an anodic onset potential of $-0.4 \mathrm{~V}$, where back-oxidation leads to electrocyclization reforming the neutral closed $( \pm)-\mathbf{1 b}-\mathbf{C}$. This is in accordance with our observation in spectroelectrochemistry (scan rate: $10 \mathrm{mV} \mathrm{s}^{-}$ 1) where no absorption of a transient radical anion species arises above $550 \mathrm{~nm}$ upon reduction (Figure 6c). Analogous to the diketone, the absorption spectrum of the closed form $( \pm)-\mathbf{1 b}-\mathbf{C}$ is fully recovered after anodic oxidation (Figure 6d). We further discuss the detailed mechanisms of the ongoing electrochemical processes at different sweep rates in the Supporting Information (Section S9).
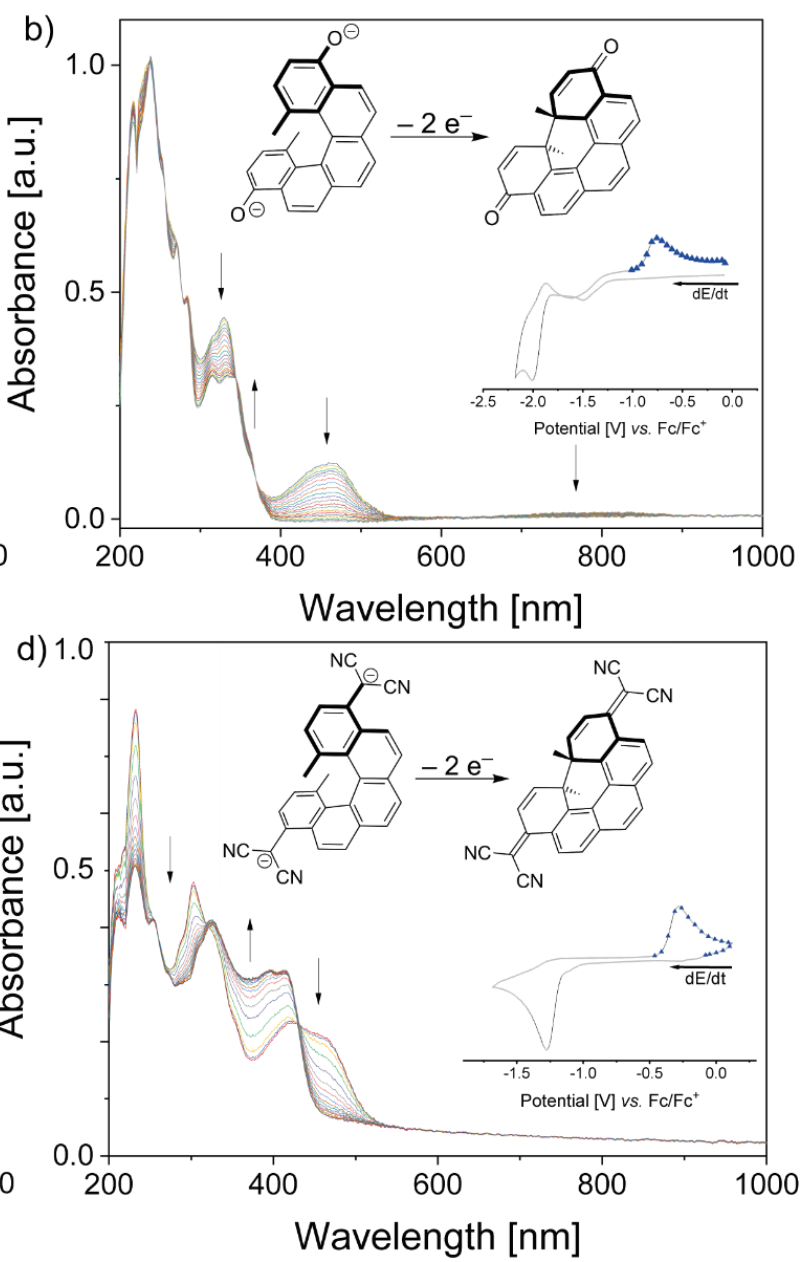

Figure 6. Spectroelectrochemistry of $( \pm)-\mathbf{1 a}-\mathbf{C}$ and $( \pm)-\mathbf{1 b}-\mathbf{C}$. a) A stable intermediate is formed upon two-electron reduction of $( \pm)-\mathbf{1 a}-\mathbf{C}, \mathrm{b})$ reoxidation of the intermediate giving back $( \pm)-\mathbf{1 a}-\mathbf{C}, \mathrm{c})$ stable intermediate formed upon two-electron reduction of $( \pm)-\mathbf{1 b}-\mathbf{C}, d)$ reoxidation of the intermediate giving back $( \pm)-\mathbf{1 b}-\mathbf{C}$. Insets showing the corresponding cyclic voltammograms with red dots or blue triangles marking when UV/Vis spectra were measured. The Experiment was carried out in MeCN with $0.1 \mathrm{M}$ $\mathrm{Bu}_{4} \mathrm{NPF}_{6}, c=6 \cdot 10^{-4} \mathrm{M}(( \pm)-\mathbf{1 a}-\mathbf{C}), c=4 \cdot 10^{-4} \mathrm{M}(( \pm)-\mathbf{1 b}-\mathbf{C}), d E / d t=10 \mathrm{mV} \mathrm{s}^{-1}$. 
Finally, we aimed for an unambiguous structural proof for the electrochemical retroelectrocyclization product under quantitative electrolysis. Coulometric reduction and subsequent oxidation in combination with HPLC analysis allowed us to determine the structure of the electrochemically generated open intermediate and the reoxidized closed form (Figure 7). The gross charge of the reductive coulometric process was determined as a twoelectron process for both, $( \pm)-\mathbf{1 a}-\mathbf{C}$ and $( \pm)-\mathbf{1 b}-\mathbf{C}$, with a quantified $78 \%$ and $72 \%$ reoxidation, respectively. Taking aliquots of the intermediate quantitative reduction product for subsequent HPLC analysis with a protic mobile phase confirmed the formation of the open form $( \pm)-\mathbf{1} \mathbf{b}-\mathbf{0}-\mathbf{H}_{2}$ by comparison with the analytical data of an authentic isolated sample obtained from the stepwise synthesis towards ( \pm )1b-C (HPLC retention time, see Figure 7, and UV/Vis absorption spectra, Figures S51, S52). The oxidative coulometric recovery of $( \pm)-\mathbf{1 b}-\mathbf{C}$ was again confirmed using the same methods. For the reduction of diketone $( \pm)$ 1a-C, no open intermediate could be trapped due to the spontaneous occurrence of electrocyclization analogously to Scheme 1. Nevertheless, full reversibility of the coulometric reduction and reoxidation to $( \pm)-\mathbf{1 a}-\mathbf{C}$ was confirmed by HPLC analysis (Figure S50). In both coulometric experiments with $( \pm)-\mathbf{1 a}-\mathbf{C}$ and $( \pm)-\mathbf{1 b}-\mathbf{C}$, no significant sideproducts were observed.

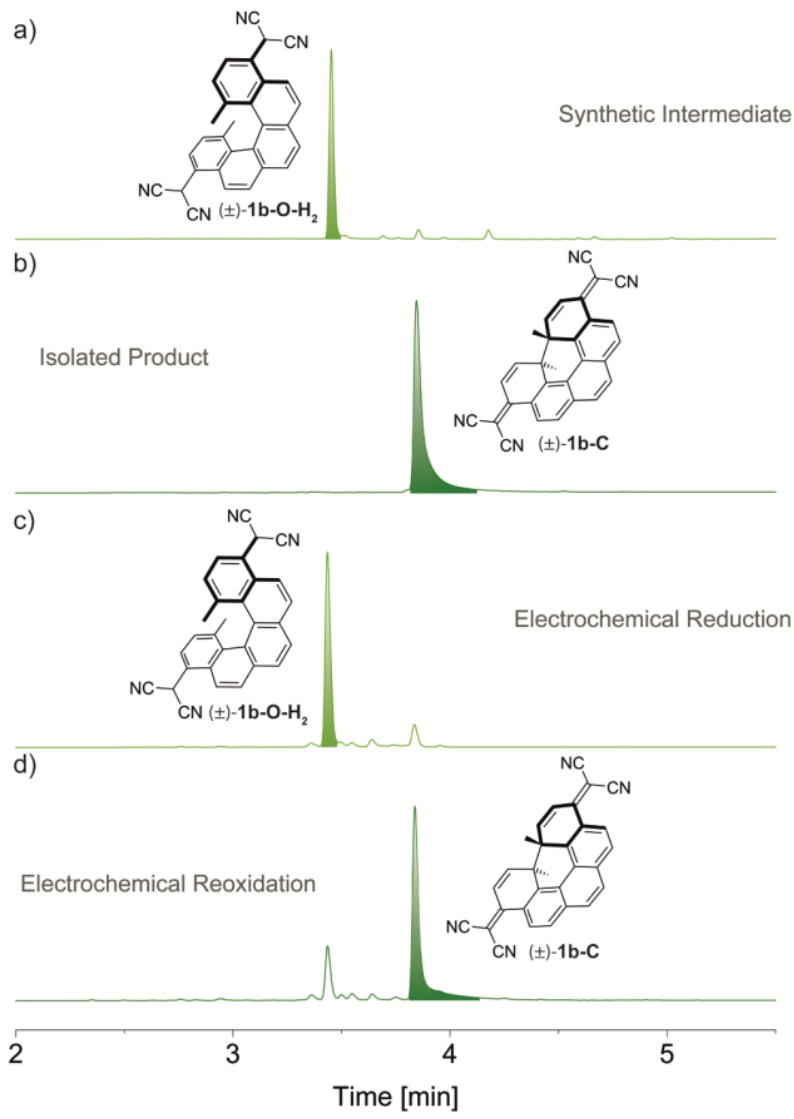

Figure 7. HPLC chromatograms of a) an authentic sample of $( \pm)-\mathbf{1 b}-\mathbf{0}-\mathbf{H}_{2}$ obtained as synthetic intermediate (Scheme 1), b) an authentic sample of $( \pm)$-1 b-C isolated during preparation, c) $( \pm)-\mathbf{1 b}-\mathbf{0}-\mathbf{H}_{2}$ obtained from (nearly) quantitative electrolysis of $( \pm)-\mathbf{1 b}-\mathbf{C}$ and protic quenching, and d) $( \pm)-\mathbf{1 b}-\mathbf{C}$ after coulometric reduction and reoxidation starting from $( \pm)-\mathbf{1 b}-\mathbf{C}$.

\section{CONCLUSION}

We demonstrated the concept of photochemical spin state switching between a diamagnetic EPR silent and a paramagnetic EPR active form of a dimethyl[5]helicene scaffold by installing radical-stabilizing substituents in the 4,11-positions. Photochemical retroelectrocyclization of the singlet closed form 1-C at cryogenic temperatures gave rise to an open-shell diradicaloid form 1-0 with a triplet ground state. The favored diradicaloid character in 1-0 is established through aromatization of the helicene core and stabilization of the radical spin density at the 4,11-oxo substituents in 1a-0 or the 4,11-dicyanomethylidene substituents in $\mathbf{1 b}-\mathbf{0}$ to avoid an energetically unfavored quinoidal closed-shell configuration. While the EPR-active triplet state of the open form is stable at cryogenic temperatures, we found that this process is fully reversible through a thermal back reaction upon heating the systems to distinct temperatures that induce the formal electrocyclization to the EPR-inactive closed form 1-C. The process can be monitored by UV/vis spectroscopy, EPR spectroscopy, and it can be even induced electrochemically via reduction of $\mathbf{1}(\mathbf{a} / \mathbf{b})-\mathbf{C}$ to the open dianions $\mathbf{1}(\mathbf{a} / \mathbf{b})-\mathbf{0}^{2-}$. Unambiguous structural characterization of the electrochemically produced open form $\mathbf{1 b}-\mathbf{O}^{2-}$ was achieved through protic quenching and HPLC analysis of $\mathbf{1 b}-\mathbf{O}-\mathbf{H}_{\mathbf{2}}$ during a quantitative electrolysis experiment. Our findings are supported by CASSCF calculations that revealed the energetic level and the diracaloid character indices of the closed- and open-shell states of each form participating in the switching. This system establishes the concept of photoswitching between different bistable spin states through chemical reactivity - unrelated to conformational stabilization - induced by light and thermal energy. We envision that the presented 4,11-substitution pattern of radical-stabilizing groups in conjunction with the 1,14dimethyl groups at the [5] helicene core has the potential for tuning various parameters of this spin state switch system through a wide range of future derivatizations. ${ }^{25}$ Towards this end, our laboratory will further report on substituent effects in various patterns and their influence towards favored spin states, suppressing the thermal back reaction and increasing the temperature of bistable operation in due course. The enantiopure intermediates $(M / P)-2$ afford synthetic access to exploit future chiroptical spin state switching and chiral recognition for sensing applications with an additional information bit.49, 50 The presented concept of induced spin state switching through bond breaking and making could serve the future demand on single-molecule magnets for applications in spintronics, data storage devices, and quantum computing. ${ }^{51,52}$ 


\section{SUPPORTING INFORMATION}

The Supporting Information is available via ChemRxiv.

\section{CCDC Accession Codes}

CCDC 2022746, 2104623, and 2129548 contain the supplementary crystallographic data for this paper. These data can be obtained free of charge via www.ccdc.cam.ac.uk/data_request/cif, or by emailing data_request@ccdc.cam.ac.uk, or by contacting The Cambridge Crystallographic Data Centre, 12 Union Road, Cambridge CB2 1EZ, UK; fax: +44 1223336033.

\section{AUTHOR INFORMATION}

\section{Corresponding Author}

Oliver Dumele Department of Chemistry \& IRIS

Adlershof, Humboldt-Universität zu Berlin, Berlin 12489,

Germany; https://orcid.org/0000-0002-3277-6570; E-mail for 0.D.: oliver.dumele@hu-berlin.de

\section{Authors}

Konstantin Günther Department of Chemistry \& IRIS

Adlershof, Humboldt-Universität zu Berlin, Berlin 12489,

Germany; https://orcid.org/0000-0002-3874-1301

Niklas Grabicki Department of Chemistry \& IRIS

Adlershof, Humboldt-Universität zu Berlin, Berlin 12489,

Germany; https://orcid.org/0000-0001-5724-7926

Beatrice Battistella Department of Chemistry \& IRIS

Adlershof, Humboldt-Universität zu Berlin, Berlin 12489,

Germany; https://orcid.org/0000-0002-5963-3684

Lutz Grubert Department of Chemistry \& IRIS

Adlershof, Humboldt-Universität zu Berlin, Berlin 12489,

Germany.

\section{Author Contributions}

$\$$ K.G. and N.G. contributed equally to this work and either has the right to list himself first in bibliographic documents. All authors have given approval to the final version of the manuscript.

\section{Funding Sources}

N.G. was supported by a doctoral fellowship of the Fonds der Chemischen Industrie (FCI) and O.D. thanks the FCI for a generous Liebig scholarship. This work was supported by a Joint Seed Fund between the Humboldt-Universität zu Berlin and the Universität Zürich, Switzerland.

\section{Notes}

The authors declare no competing financial interest.

\section{ACKNOWLEDGMENT}

We are indebted to Michal Juríček (Univ. Zürich) and his research group who served as great inspiration for this work and contributed with fruitful discussions. We thank David Furkert (Leibniz-Institut für Molekulare Pharmakologie, Berlin) and Sven Herter for measuring HRMS, Minh Bui for assistance with IR spectroscopy, Kristin Klaue for helpful discussions, and Björn Kobin for developing the synthetic irradiation setup. We are grateful to Stefan Hecht for his continuous support.

\section{REFERENCES}

(1) Itkis, M.; Chi, X.; Cordes, A.; Haddon, R. Magneto-Opto-Electronic Bistability in a Phenalenyl-Based Neutral Radical. Science 2002, 296 (5572), 1443-1445.

(2) Wolf, S.; Awschalom, D.; Buhrman, R.; Daughton, J.; von Molnár, S.; Roukes, M.; Chtchelkanova, A. Y.; Treger, D. Spintronics: A SpinBased Electronics Vision for the Future. Science 2001, 294 (5546), 1488-1495.

(3) Sanvito, S. Molecular spintronics. Chem. Soc. Rev. 2011, 40 (6), 3336-3355. 
(4) Bogani, L.; Wernsdorfer, W. Molecular spintronics using single-molecule magnets. Nat. Mat. 2008, 7, $194-201$.

(5) Goulet-Hanssens, A.; Eisenreich, F.; Hecht, S. Enlightening Materials with Photoswitches. Adv. Mater. 2020, 32 (20), 1905966.

(6) Fuchter, M. J. On the Promise of Photopharmacology Using Photoswitches: A Medicinal Chemist's Perspective. J. Med. Chem. 2020, 63 (20), 11436-11447.

(7) Bayliss, S.; Laorenza, D.; Mintun, P.; Kovos, B.; Freedman, D. E.; Awschalom, D. Optically addressable molecular spins for quantum information processing. Science 2020, 370 (6522), 1309-1312.

(8) Nakazawa, S.; Nishida, S.; Sato, K.; Toyota, K.; Shiomi, D.; Morita, Y.; Sugisaki, K.; Hosseini, E.; Maruyama, K.; Yamamoto, S., Molecular Spin Qubits: Molecular Optimization of Synthetic Spin Qubits, Molecular Spin AQC and Ensemble Spin Manipulation Technology. In Principles and Methods of Quantum Information Technologies, Springer: 2016; pp 605-624.

(9) Sato, O.; Tao, J.; Zhang, Y. Z. Control of Magnetic Properties through External Stimuli. Angew. Chem. Int. Ed. 2007, 46 (13), $2152-2187$.

(10) Frisenda, R.; Harzmann, G. D.; Celis Gil, J. A.; Thijssen, J. M.; Mayor, M.; Van Der Zant, H. S. Stretching-Induced Conductance Increase in a Spin-Crossover Molecule. Nano Lett. 2016, 16 (8), 4733-4737.

(11) Harzmann, G. D.; Frisenda, R.; van der Zant, H. S.; Mayor, M. Single-Molecule Spin Switch Based on Voltage-Triggered Distortion of the Coordination Sphere. Angew. Chem. Int. Ed. 2015, 54 (45), 13425-13430.

(12) Ishiguro, K.; Ozaki, M.; Sekine, N.; Sawaki, Y. Acid-Base Equilibrium between Phenoxyl-Nitronyl Nitroxide Biradical and Closed-Shell Cation. A Magnetic pH Sensor. J. Am. Chem. Soc. 1997, 119 (15), 3625-3626.

(13) Venkataramani, S.; Jana, U.; Dommaschk, M.; Sönnichsen, F.; Tuczek, F.; Herges, R. Magnetic Bistability of Molecules in Homogeneous Solution at Room Temperature. Science 2011, 331 (6016), 445-448.

(14) Milek, M.; Heinemann, F. W.; Khusniyarov, M. M. Spin Crossover Meets Diarylethenes: Efficient Photoswitching of Magnetic Properties in Solution at Room Temperature. Inorg. Chem. 2013, 52 (19), 11585-11592.

(15) Hayami, S.; Holmes, S. M.; Halcrow, M. A. Spin-state switches in molecular materials chemistry. J. Mat. Chem. C 2015, 3 (30), 77757778.

(16) Tanaka, K.; Toda, F. A novel photochromism of biindenylidene in crystal form. J. Chem. Soc., Perkin Trans. 1 2000, (6), 873-874.

(17) Kurata, H.; Kim, S.; Matsumoto, K.; Kawase, T.; Oda, M. Photochemical and Redox Switching in a Ring-closed Isomer of Thioindigoextended quinone. Chem. Lett. 2007, 36 (3), 386-387.

(18) Nishiuchi, T.; Ito, R.; Stratmann, E.; Kubo, T. Switchable Conformational Isomerization of an Overcrowded Tristricyclic Aromatic Ene. J. Org. Chem. 2019, 85 (1), 179-186.

(19) Wonink, M. B.; Corbet, B. P.; Kulago, A. A.; Boursalian, G. B.; De Bruin, B.; Otten, E.; Browne, W. R.; Feringa, B. L. Three-State Switching of an Anthracene Extended Bis-thiaxanthylidene with a Highly Stable Diradical State. J. Am. Chem. Soc. 2021, 143 (43), 18020-18028.

(20) Nakatsuji, S. Recent progress toward the exploitation of organic radical compounds with photo-responsive magnetic properties. Chem. Soc. Rev. 2004, 33 (6), 348-353.

(21) Šolomek, T.; Ravat, P.; Mou, Z.; Kertesz, M.; Juríček, M. Cethrene: The Chameleon of Woodward-Hoffmann Rules. J. Org. Chem. 2018, $83(8), 4769-4774$.

(22) Ravat, P.; Hinkelmann, R.; Steinebrunner, D.; Prescimone, A.; Bodoky, I.; Juríček, M. Configurational Stability of [5]Helicenes. Org. Lett. 2017, 19 (14), 3707-3710.

(23) Ishiyama, T.; Murata, M.; Miyaura, N. Palladium(0)-Catalyzed Cross-Coupling Reaction of Alkoxydiboron with Haloarenes: A Direct Procedure for Arylboronic Esters. J. Org. Chem. 1995, 60 (23), 7508-7510.

(24) Ravat, P.; Šolomek, T.; Rickhaus, M.; Häussinger, D.; Neuburger, M.; Baumgarten, M.; Juríček, M. Cethrene: A Helically Chiral Biradicaloid Isomer of Heptazethrene. Angew. Chem. Int. Ed. 2016, 55 (3), 1183-1186.

(25) Ravat, P.; Šolomek, T.; Häussinger, D.; Blacque, O.; Juríček, M. Dimethylcethrene: A Chiroptical Diradicaloid Photoswitch. J. Am. Chem. Soc. 2018, 140 (34), 10839-10847.

(26) Uno, M.; Seto, K.; Takahashi, S. A new method of synthesis of arylmalononitriles catalysed by a palladium complex. J. Chem. Soc., Chem. Commun. 1984, (14), 932-933.

(27) Woodward, R. B.; Hoffmann, R. The Conservation of Orbital Symmetry. Angew. Chem. Int. Ed. 1969, 8 (11), $781-853$.

(28) Steiner, R. P.; Miller, R. D.; Dewey, H. J.; Michl, J. Photochemical generation of 2, 3-naphthoquinodimethane derivatives. An extremely facile" forbidden" thermal reaction and evidence for a low-energy doubly excited state. J. Am. Chem. Soc. 1979, 101 (7), $1820-1826$.

(29) Solà, M. Forty years of Clar's aromatic $\pi$-sextet rule. Front. Chem. 2013, 1, 22.

(30) Clar, E.; Kemp, W.; Stewart, D. The significance of Kekulé structures for the stability of aromatic systems. Tetrahedron 1958, 3 (3), $325-333$.

(31) Clar, E.; Macpherson, I. A. The significance of Kekulé structures for the stability of aromatic systems-II. Tetrahedron 1962, 18 (12), $1411-1416$

(32) Sun, Z.; Lee, S.; Park, K. H.; Zhu, X.; Zhang, W.; Zheng, B.; Hu, P.; Zeng, Z.; Das, S.; Li, Y. Dibenzoheptazethrene Isomers with Different Biradical Characters: An Exercise of Clar's Aromatic Sextet Rule in Singlet Biradicaloids. J. Am. Chem. Soc. 2013, 135 (48), 18229 18236.

(33) Yamaguchi, K. The electronic structures of biradicals in the unrestricted Hartree-Fock approximation. Chem. Phys. Lett. 1975, 33 (2), 330-335.

(34) Valenta, L.; Mayländer, M.; Kappeler, P.; Blacque, O.; Šolomek, T.; Richert, S.; Juríček, M. Validation of the triplet ground state in a persistent derivative of Clar's hydrocarbon. Chem. Commun., DOI: 10.1039/D2CC00352J.

(35) Dressler, J. J.; Zhou, Z.; Marshall, J. L.; Kishi, R.; Takamuku, S.; Wei, Z.; Spisak, S. N.; Nakano, M.; Petrukhina, M. A.; Haley, M. M. Synthesis of the Unknown Indeno [1,2-a]fluorene Regioisomer: Crystallographic Characterization of Its Dianion. Angew. Chem. Int. Ed. 2017, 129 (48), 15565-15569.

(36) Zeng, Z.; Sung, Y. M.; Bao, N.; Tan, D.; Lee, R.; Zafra, J. L.; Lee, B. S.; Ishida, M.; Ding, J.; Lopez Navarrete, J. T. Stable TetrabenzoChichibabin's Hydrocarbons: Tunable Ground State and Unusual Transition between Their Closed-Shell and Open-Shell Resonance Forms. J. Am. Chem. Soc. 2012, 134 (35), 14513-14525.

(37) Gallagher, N.; Zhang, H.; Junghoefer, T.; Giangrisostomi, E.; Ovsyannikov, R.; Pink, M.; Rajca, S.; Casu, M. B.; Rajca, A. Thermally and Magnetically Robust Triplet Ground State Diradical. J. Am. Chem. Soc. 2019, 141 (11), 4764-4774.

(38) Wang, Z. Y.; Dai, Y. Z.; Ding, L.; Dong, B. W.; Jiang, S. D.; Wang, J. Y.; Pei, J. A Stable Triplet-Ground-State Conjugated Diradical Based on a Diindenopyrazine Skeleton. Angew. Chem. Int. Ed. 2021, 60 (9), 4594-4598.

(39) Abe, M. Diradicals. Chem. Rev. 2013, 113 (9), 7011-7088.

(40) Karafiloglou, P. The double (or dynamic) spin polarization in $\pi$ diradicals. J. Chem. Educ. 1989, 66 (10), 816. 
(41) Hsieh, Y.-C.; Wu, C.-F.; Chen, Y.-T.; Fang, C.-T.; Wang, C.-S.; Li, C.-H.; Chen, L.-Y.; Cheng, M.-J.; Chueh, C.-C.; Chou, P.-T. 5,14Diaryldiindeno $\left[2,1-f: 1^{\prime}, 2^{\prime}-j\right]$ picene: A New Stable [7]Helicene with a Partial Biradical Character. J. Am. Chem. Soc. 2018, 140 (43), $14357-14366$.

(42) Koshido, T.; Kawai, T.; Yoshino, K. Optical and Electrochemical Properties of cis-1,2-Dicyano-1,2-bis(2,4,5-trimethyl-3-thienyl)ethene. J. Phys. Chem. 1995, 99 (16), 6110-6114.

(43) Peters, A.; Branda, N. R. Electrochromism in Photochromic Dithienylcyclopentenes. J. Am. Chem. Soc. 2003, 125 (12), $3404-3405$.

(44) Browne, W. R.; de Jong, J. J.; Kudernac, T.; Walko, M.; Lucas, L. N.; Uchida, K.; van Esch, J. H.; Feringa, B. L. Oxidative electrochemical switching in dithienylcyclopentenes, part 1: effect of electronic perturbation on the efficiency and direction of molecular switching. Chem. Eur. J. 2005, 11 (21), 6414-6429.

(45) Browne, W. R.; de Jong, J. J.; Kudernac, T.; Walko, M.; Lucas, L. N.; Uchida, K.; van Esch, J. H.; Feringa, B. L. Oxidative electrochemical switching in dithienylcyclopentenes, part 2: effect of substitution and asymmetry on the efficiency and direction of molecular switching and redox stability. Chem. Eur. J. 2005, 11 (21), 6430-6441.

(46) Staykov, A.; Areephong, J.; Browne, W. R.; Feringa, B. L.; Yoshizawa, K. Electrochemical and Photochemical Cyclization and Cycloreversion of Diarylethenes and Diarylethene-Capped Sexithiophene Wires. ACS Nano 2011, 5 (2), 1165-1178.

(47) Moriyama, Y.; Matsuda, K.; Tanifuji, N.; Irie, S.; Irie, M. Electrochemical Cyclization/Cycloreversion Reactions of Diarylethenes. Org. Lett. 2005, 7 (15), 3315-3318.

(48) Evans, D. H.; Hu, K. Inverted potentials in two-electron processes in organic electrochemistry. J. Chem. Soc., Faraday Trans. 1996, 92 (20), 3983-3990.

(49) Schweinfurth, D.; Zalibera, M.; Kathan, M.; Shen, C.; Mazzolini, M.; Trapp, N.; Crassous, J.; Gescheidt, G.; Diederich, F. Helicene Quinones: Redox-Triggered Chiroptical Switching and Chiral Recognition of the Semiquinone Radical Anion Lithium Salt by Electron Nuclear Double Resonance Spectroscopy. J. Am. Chem. Soc. 2014, 136 (37), 13045-13052.

(50) Teichmann, B.; Krause, A.-M.; Lin, M.-J.; Würthner, F. Enantioselective Recognition of Helicenes by a Tailored Chiral

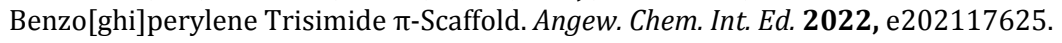

(51) Troiani, F.; Affronte, M. Molecular spins for quantum information technologies. Chem. Soc. Rev. 2011, 40 (6), 3119-3129.

(52) Sato, K.; Nakazawa, S.; Rahimi, R.; Ise, T.; Nishida, S.; Yoshino, T.; Mori, N.; Toyota, K.; Shiomi, D.; Yakiyama, Y. Molecular electronspin quantum computers and quantum information processing: pulse-based electron magnetic resonance spin technology applied to matter spin-qubits. J. Mater. Chem. 2009, 19 (22), 3739-3754. 